CERN-PPE/95-152

September 21, 1995

\title{
Beam Test Results of a Shashlik Calorimeter in High Magnetic Field
}

\section{RD36 Collaboration}

P. Aspell, S. Bates, Ph. Bloch, R. Grabit, P. Jarron, F. Lemeilleur, R. Loos, A. Marchioro, E. Rosso CERN, Geneva, SWITZERLAND

J. Badier, Ph. Bruel, A. Busata, Ph. Busson, C. Charlot, F. Dorchies, L. Dobrzynski, O. Ferreira, Ch. Gregory, A. Karar, P. Manigot, R. Tanaka, J.Ch. Vanel LPNHE, Ecole Polytechnique, Palaiseau, FRANCE

S. Bityukov, V. Obraztsov, A. Ostankov, Yu. Protopopov, V. Rykalin, P. Spiridonov, V. Soushkov, V. Vasil'chenko IHEP, Protvino, RUSSIA

E. Clayton, D. Miller, C. Seez, T.S. Virdee

Imperial College, London, UK

R. Djilkibaev, S. Gninenko, E. Guschin, Y. Musienko, V. Popov, A. Skasyrskaya, I. Semenyuk INR, Moscow, RUSSIA

I. Cheremukhin, A. Egorov*), I. Golutvin, Y. Kozlov*), P. Moissenz, S. Sergueev, A. Sidorov*), E. Zubarev, N. Zamiatin JINR, Dubna, RUSSIA

P. Bordalo, S. Ramos, J. Varela LIP, Lisboa, PORTUGAL

D.J.A. Cockerill, J. Connolly, L. Denton Rutherford Appleton Laboratory, Didcot, UK

N. Godinovic, I. Puljak, I. Soric

FESB, University of Split, Split, CROATIA

S.R. Chendvankar, S.K. Gupta, S.N. Ganguli, A. Gurtu, M. Maity, G. Majumder, K.Mazumdar, T. Moulik

EHEP Group, Tata Institute of Fundamental Research, Colaba, Bombay, INDIA

*) Elma Industry, RUSSIA 


\section{Abstract}

Shashlik calorimeter prototypes equipped with preshower detector have been tested in 3 Tesla magnetic field with electron beam at CERN-SPS. The signal from electrons increases as much as $11 \%$ at 3 Tesla magnetic field. No significant deterioration on the energy resolution as well as the preshower detector performance have been observed.

(To be submitted to Nuclear Instruments and Methods) 
In the design of the CMS detector at LHC [1,2], the electromagnetic calorimeter operates inside a strong magnetic field (4 Tesla). An option for the CMS detector is a lead/scintillator sandwich sampling calorimeter read by wave-length-shifting (WLS) fibres, called "Shashlik" [3, 4]. We have tested a prototype of projective towers assembled in a $3 \times 5$ matrix, including a preshower detector, in the 3 Tesla field generated by the EHS magnet at the SPS-H2 beam line at CERN during April-May 1994.

The experimental setup is described in Section 2. Signal response in a magnetic field is discussed in Section 3. The energy resolution with/without preshower detector will be discussed in Section 4.

\section{$2 \quad$ Experimental Setup}

The schematic view of the beam test setup is shown in Fig. 1. The Shashlik towers were assembled as a $3 \times 5$ matrix. The central tower of this matrix was exposed to $15-150 \mathrm{GeV}$ electron beams to study the energy resolution response.

\subsection{Shashlik and preshower detector}

The projective prototypes studied here were constructed in 1993. The basic parameters are given in the Table 1 . These towers have a truncated pyramid shape, and consist of 75 layers of lead $(2 \mathrm{~mm})$ and scintillators $(4 \mathrm{~mm})$. The scintillation light is readout via $6 \times 6$ matrix of WLS fibres which are perpendicular to the plates. The fibres themselves are in parallel each other, thus the distance between the outermost set of fibres and the scintillator edges will increase for deeper position. The WLS fibres are curved as U-shape in front of the calorimeter, and are bundled into a silicon PIN photodiode (Hamamatsu S3590-05) with an area of $1 \times 1 \mathrm{~cm}^{2}$. The silicon photodiode and the charge amplifier are insensitive to the magnetic field. The signals have been readout by LeCroy 2282A (12 bits) ADC's. The beam test results, in the absence of magnetic field, are reported in previous papers $[5,6]$. The light yield of these towers is measured to be 12 photons $/ \mathrm{MeV}$.

The preshower detector contains 2 planes of silicon strip detectors. Each plane is built out of 4 wafers $\left(6 \times 6 \mathrm{~cm}^{2}\right.$ each), with $2 X_{0}$ and $1 X_{0}$ absorber $(\mathrm{Pb})$ in front, respectively. The pitch of the strips is $2 \mathrm{~mm}$. The strips have been oriented orthogonally in the two planes. The signals were readout by a 16-channel AMPLEX-SiCAL signal processor [7]. Each detector was connected to a printed board circuit containing two AMPLEX's.

\subsection{SPS-H2 beam line}

The trigger is generated by the coincidence of three scintillation counters, $\mathrm{S} 1(10 \times$ $\left.10 \times 0.5 \mathrm{~cm}^{3}\right)$, S4H $\left(2 \times 2 \times 1 \mathrm{~cm}^{3}\right)$ and $\mathrm{S} 4\left(4 \times 4 \times 1 \mathrm{~cm}^{3}\right)$. The tracking of the particle is obtained with two delayline wire-chambers (DWC), which had $300 \mu \mathrm{m}$ intrinsic resolution [8].

Based on the experience of previous beam tests, special attention has been paid to reduce the amount of material in front of the calorimeter as the energy resolution is degraded due to bremsstrahlung. This is especially important for tests in a magnetic field. While the major contribution arose from the three scintillation trigger counters $\left(5.9 \% X_{0}\right)$, the thickness of air was not negligible $\left(2.6 \% X_{0}\right)$. The total amount of material is $9.8 \% X_{0}$. Monte Carlo simulation shows that this extra material does not induce a significant tail in the energy resolution. 


\subsection{The EHS magnetic field}

The Shashlik and preshower detectors have been placed at the center of EHS magnet [9]. The magnetic field map is shown in Fig. 2. The beam direction is taken along the $z$-axis. The field is along the $x$-axis, perpendicular to the beam. The largest side of the Shashlik $3 \times 5$ matrix is then in the bending direction $(y$-axis). The peak field value is 3 Tesla, and the full bending power is $\int B d l=5.7 \mathrm{Tm}$. The Shashlik matrix center was placed at the EHS magnet center $(x=y=z=0)$. The fully equipped Shashlik tower is about $65 \mathrm{~cm}$ long and the entrance is at $z=-30 \mathrm{~cm}$. When the preshower detector is installed, the beam impact point (at the front surface of the first radiator material of $\left.2 X_{0}\right)$ is at $z=-35 \mathrm{~cm}$.

Monte Carlo simulations show that due to the transverse magnetic field it is important to have a compact preshower detector, i.e. to keep the distance from the lead plate and the following silicon layer as small as possible (much less than $1 \mathrm{~cm}$ ). The distance between preshower detector and Shashlik must also be kept small to avoid any shower leakage due to low energy electron/positrons which are swept away by the strong magnetic field.

\section{$3 \quad$ Shashlik and Preshower Detector in Magnetic Field}

As the Shashlik calorimeter is intended to operate in the strong CMS magnetic field, it is important to study its possible consequences on the electromagnetic shower energy measurement. The signal distribution, the energy resolution together with longitudinal and transverse profiles are studied. The central tower of the Shashlik matrix is used to study energy resolution in a $0-3$ Tesla magnetic field with and without the preshower detector.

\subsection{Shashlik response in magnetic field}

The Shashlik energy response without the preshower detector is shown in Fig. 3 at 0,1 and 3 Tesla for $150 \mathrm{GeV}$ electrons. One sees a displacement of the peak when the field increases. The tail on the high-energy side is due to the "nuclear counter effect" in the silicon photodiode due to shower leakage from the rear of the calorimeter. Charged particles traversing the PIN photodiode leave an energy equivalent of a few $\mathrm{GeV}$ due to ionization energy loss. In addition, there can be some shower leakage via the holes in the scintillator plates not converted by the WLS fibre. Cerenkov light in WLS fibres may also contribute to the non-Gaussian tail.

The response in the magnetic field for Shashlik alone or with $3 X_{0}$ of passive material in front is plotted in Fig. 4 as function of the magnetic field. The calibration constants were kept at the values obtained at 0 Tesla. The response for $150 \mathrm{GeV}$ electrons is obtained by summing signals in 9 towers. Parametrizing the light increase as $S=S_{0} \times(1+\alpha \sqrt{ } B)$, we can extrapolate to the 4 Tesla CMS field where we expect a signal increase of about $13 \%$ compared to 0 Tesla. Such an increase will have to be taken into account for the calibration of the Shashlik modules when used in the magnetic field. When there is passive material $\left(3 X_{0}\right)$ in front of calorimeter, the variation is slightly smaller. The reason is probably due to low energy electrons/positrons which are stopped or swept before reaching the Shashlik detector.

Using pedestal and test pulse data, we can exclude the possibility that the change in response is due to a variation of the gain of the readout system. On the other hand, one may expect a modification of the electron sampling fraction due to the curvature of low energy electrons in the field. A Monte Carlo simulation (GEANT 3.21) of the 
full sampling structure has been performed down to a cutoff of $10 \mathrm{keV}$ for the energy of electrons and gammas. The result is presented in Fig. 5 where signal distributions for $1 \mathrm{GeV}$ electrons without and with a 4 Tesla transverse field are shown. The difference in mean signal response between 0 and 4 Tesla data is less than $1 \%$. Similar results have been obtained for $10 \mathrm{GeV}$ electrons as well. We then conclude that the observed signal increase is due to a light yield increase. Such an effect has already been reported [10] for SCSN-38 Kuraray type scintillator, although at a lower field intensity (1.5 Tesla). A detailed study concluded that this phenomenon can probably be explained by the effect of magnetic field on base molecules excitation or energy transfer to the first fluor in the scintillator [11].

The energy resolution at $150 \mathrm{GeV}$ is listed in Table 2 for 3 different field values. It is found that the resolution is independent of the field. Similar results are obtained with the preshower detector in front of the calorimeter.

\subsection{Shashlik signal linearity}

To study the linearity and the intrinsic energy resolution, a correction for lateral non-uniformity was applied to the data [12]. The global response is corrected with the 2nd order polynomial function. The local fibre effect due to Čerenkov light or due to shower leakage around the fibres is also corrected with a cos-wave function superimposed to the polynomial. The linearity of response in 0 and 3 Tesla is shown in Fig. 6 . In the absence of magnetic field, the linearity is better than $\pm 0.2 \%$. In magnetic field, the linearity at low energy is poor. Low energy electrons hit the lower boundary region with a large incident angle so that the electromagnetic shower is shared almost equally between the central tower and the lower adjacent tower of the Shashlik matrix. Due to the air gap between these two towers $(\sim 500 \mu \mathrm{m})$, part of the energy is lost. Although we find a slightly better linearity with a shower clustering in a larger zone $(3 \times 4$ instead of $3 \times 3)$, we observed exactly the same energy resolution with the $3 \times 3$ and the $3 \times 4$ clustering method.

\subsection{Effect of rear leakage}

As seen in Fig. 3, we observe for high energy showers an excess of events above the peak value. Such a high energy tail is due to charged particles leaking out at the rear of the Shashlik modules (27 $X_{0}$ long) and giving a signal in the photodiode (see also ref.[5]). This tail has been studied as a function of the calorimeter depth and of the field intensity. The excess of events is defined as the fraction of events that give a signal greater than $2 \sigma$ above the peak value. The results are shown in Fig. 7. The points at $30 X_{0}$ correspond to data with the preshower detector in front of the Shashlik. We observe that the tail is reduced, as expected, when increasing the calorimeter depth, but also by a factor three (for $27 X_{0}$ ) or six (for $30 X_{0}$ ) when going from 0 to 3 Tesla magnetic field. This can be understood by the trapping in the field of the low energy electrons exiting the rear of the Shashlik tower.

\subsection{Effect on transverse profile}

The position resolution without magnetic field has already been reported elsewhere [5]. In the magnetic field, one may expect a deformation of the shower profile and therefore a modification of the position resolution.

In Fig. 8 is presented the transverse profile for $80 \mathrm{GeV}$ electrons in the direction parallel to the field. The energy in each tower is normalised to the sum of energies deposited in 9 towers and expressed in percent, in order to unfold the effect of increase in 
light. Monte Carlo predictions are also shown in Fig. 8 and are in good agreement with data. The precision of the simulation is of the order of $\pm 1 \%$. This uncertainty is due to the incomplete description of the exact tower geometry, and of the beam impact point and profile. As one can see from data points, the electromagnetic shower slightly shrinks in field direction.

The transverse profile in the direction perpendicular to the field is presented in Fig. 9 for $\mathrm{B}=0$ and 3 Teslas. The data in 0 Tesla is asymmetric due to the position of the incidence point of the beam being at $-8 \mathrm{~mm}$ with respect to tower center. An increase of the lateral spread of the shower in the field is observed in the data and is well reproduced by Monte Carlo simulation. It appears to be symmetrical and amounts to a $40 \%$ increase of energy in towers neigbouring the central one. This implies that the dominant effect of the field on the shower is on electrons/positrons pairs.

The position resolution is shown in Fig. 10, without and with magnetic field, as a function of the distance to tower center. No significant difference in position resolution between the two configurations (with/without preshower detector) is observed for 0 Tesla. A resolution of $1.5 \mathrm{~mm}$ is achieved at the tower center and $0.5 \mathrm{~mm}$ at the tower edge. The resolution obtained with field is slightly better (by $\sim 15 \%$ ) than with field off, due to the modification of the energy sharing between central and next to central towers.

\section{$4 \quad$ Energy Resolution}

\subsection{Bare Shashlik}

The energy resolution of the calorimeter is generally parametrized as,

$$
\frac{\sigma_{E}}{E}=\frac{a}{\sqrt{E}} \oplus \frac{b}{E} \oplus c
$$

where $a$ represents the stochastic term, $b$ the electronics noise term, $c$ the constant term, and $E$ is the energy in $\mathrm{GeV}$.

The noise term has been studied using the width of the pedestals. No significant correlated noise between readout channels has been observed, i.e. the equivalent noise per channel was equal to the noise $b$ divided by three for $3 \times 3$ clustering. The noise is equivalent to $173 \mathrm{MeV}$ per channel for 0 Tesla data. No change in noise is observed when magnet is on, and is $159 \mathrm{MeV}$ per channel for 3 Tesla data. This virtual improvement is due to the light output increase in the magnetic field. When fitting the energy resolution, the noise term, $0.519 / E$ (0 Tesla) or $0.476 / E$ (3 Tesla), is fixed and subtracted from the data.

The energy resolution of a bare Shashlik for an area of $2 \times 2 \mathrm{~cm}^{2}$ at the tower center is shown in Fig. 11. When the magnet is off (0 Tesla), the energy resolution is found to be

$$
\frac{\sigma_{E}}{E}=\frac{8.73 \%}{\sqrt{E}} \oplus 0.70 \% .
$$

In the 3 Tesla magnetic field, we measure the same resolution for energies above $40 \mathrm{GeV}$. The fit result is

$$
\frac{\sigma_{E}}{E}=\frac{8.89 \%}{\sqrt{E}} \oplus 0.62 \%
$$

For lower energies, a slightly worse resolution is observed. This is due to the fact that lower momentum electrons hit the lower part of the central tower with an incident angle of a few degrees. The energy resolution at the tower boundary region is not as good as that at the tower center due to the shower leakage between towers. 


\subsection{Shashlik + preshower detector}

The energy resolution of a Shashlik tower with the preshower detector in front is shown in Fig. 12 for a field of 3 Tesla. The energy resolution, fitted to data above $40 \mathrm{GeV}$, is given by

$$
\frac{\sigma_{E}}{E}=\frac{8.39 \%}{\sqrt{E}} \oplus 0.60 \% .
$$

Due to fiducial cuts in the preshower detector, the data correspond to a smaller area of $0.6 \times 0.8 \mathrm{~cm}^{2}$. The slightly better resolution when compared with Shashlik alone can be explained by a smaller residual non-uniformity.

\section{Conclusion}

The Shashlik and an active preshower detector have been tested with electron beams of $15-150 \mathrm{GeV} / \mathrm{c}$ in 0 and 3 Tesla magnetic field. The scintillation light yield increases in the presence of magnetic field by $+11 \%$ at 3 Tesla. No significant deterioration in the position and energy resolution has been observed at 3 Tesla. When a preshower detector is placed in front of the Shashlik, the energy resolution is measured to be $\sigma_{E} / E=8.4 \% / \sqrt{E} \oplus 0.476 / E \oplus 0.6 \%$ above $40 \mathrm{GeV}$.

\section{Acknowledgements}

We would like to thank the CERN-SPS machine crew and N. Doble for beam operation. We are grateful for the support given by the RD5 collaboration, especially by G. Bencze, F. Szoncso and C.-E. Wulz for tests in the SPS-H2 beam line. Finally, we would also like to thank B. Powell for the operation of EHS magnet.

\section{References}

[1] CMS Collaboration, Letter of Intent for a General Purpose Detector at the LHC, CERN/LHCC 92-3/I1 (1992).

[2] CMS Collaboration, Technical Proposal, CERN/LHCC 94-38 (1994).

[3] CERN-DRDC Proposal-50, "A combined Shashlik + Preshower detector for LHC", J. Badier et al., CERN/DRDC 93-28 (1993).

[4] CERN-DRDC 94-47, RD36 Status Report, Jan. 1995.

[5] J. Badier et al., "New Test Beam Results of Shashlik and Preshower Prototypes", CMS TN/94-152, INR-984/94, X-LPNHE/94-01 (1994).

[6] "Shashlik Calorimeter for CMS", in Proceeding of the IV International Conference on Calorimetry in High Energy Physics, p.158, Isola d'Elba, Italy, September 19-25, 1993.

[7] E. Beuville et al., Nucl. Instr. and Meth., A288 (1990) 157, Nucl. Phys. B (Proc. Suppl.), 23A (1991) 198, IEEE Trans. Nucl. Sci. 39 (1992) 766.

[8] A. Manarin and G. Vismara, "The Delay Wire Chamber (DWC) Description", CERN LEP/BI-TA/Note 85-3 (1985).

[9] C. Lesmond, "L'aimant M1 de EHS", SUPRA/78-05 (Saclay), and by courtesy of RD5 collaboration for EHS field mapping data.

[10] J. Mainusch et al., Nucl. Instr. and Meth., A312 (1992) 451.

[11] D. Blomker et al., IEEE Trans. Nucl. Sci. NS-37(2) (1990) 220.

[12] P. Aspell et al., "Energy and Spatial Resolution of a Shashlik Calorimeter and a Silicon Preshower Detector", CERN-PPE/95-151 (1995), to be published in NIM. 


\begin{tabular}{|l|c|}
\hline Number of towers & 16 \\
\hline Tower lateral size & $\begin{array}{c}52 \times 52 \mathrm{~mm}^{2} \text { (front) } \\
64 \times 64 \mathrm{~mm}^{2} \text { (rear) }\end{array}$ \\
\hline Number of planes & 75 \\
\hline Scintillator/Lead & $4 \pm 0.05 \mathrm{~mm} / 2 \pm 0.005 \mathrm{~mm}$ \\
\hline \hline Total radiation length & $27.5 X_{0}$ \\
\hline Radiation length & $16.9 \mathrm{~mm}$ \\
\hline Molière radius & $34 \mathrm{~mm}$ \\
\hline \hline Scintillator & polystyrene $+0.5 \%$ POPOP $+2 \%$ para-terphenyl \\
\hline WLS fibre & Kr Y7, $\phi=1.2 \mathrm{~mm}$ \\
\hline Number of fibres & 36 \\
\hline Interfibre distance & U-shape loop \\
\hline Front fibre ends & Photodiode + Amplifier \\
\hline \hline Readout
\end{tabular}

Table 1: Parameters for the Shashlik projective towers.

\begin{tabular}{|c|c||c|}
\hline $\begin{array}{c}\text { Beam Energy } \\
E_{\text {beam }}(\mathrm{GeV})\end{array}$ & $\begin{array}{c}\text { Magnetic Field } \\
B(\text { Tesla })\end{array}$ & $\begin{array}{c}\text { Energy Resolution } \\
\sigma_{E} / E(\%)\end{array}$ \\
\hline \hline 150 & 0 & $0.89 \pm 0.03$ \\
\hline 150 & 1 & $0.89 \pm 0.03$ \\
\hline 150 & 3 & $0.84 \pm 0.03$ \\
\hline \hline
\end{tabular}

Table 2: Energy resolution for $150 \mathrm{GeV}$ electrons for the magnetic field intensity of 0,1 and 3 Tesla. 


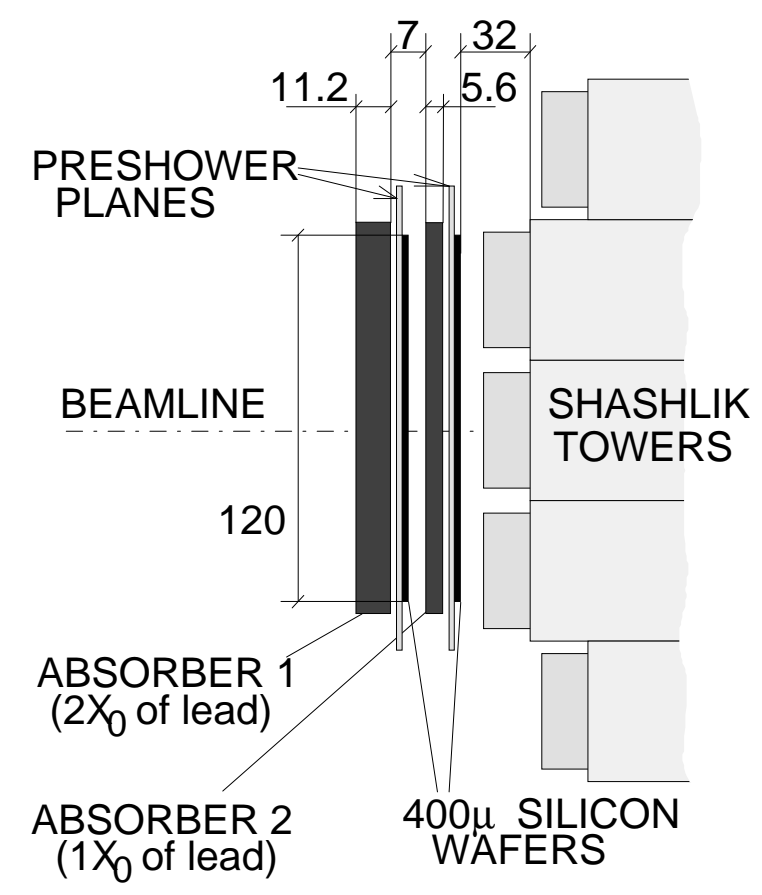

Figure 1: Preshower detector geometry for H2 beam test in 1994. 

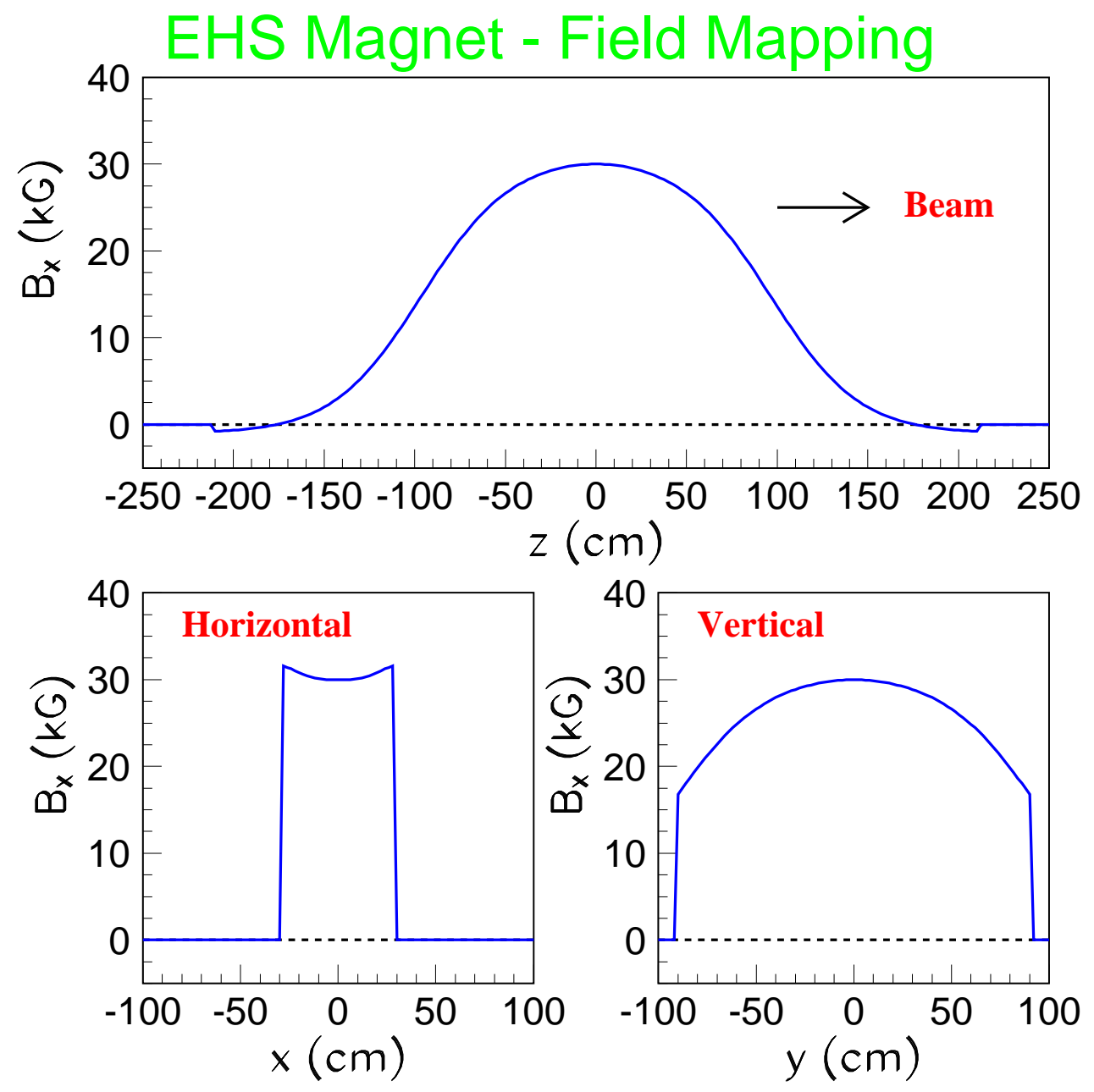

Figure 2: EHS magnet field map. The magnetic field was along $x$-axis, and the field strength $B_{x}$ is drawn along each axis. 


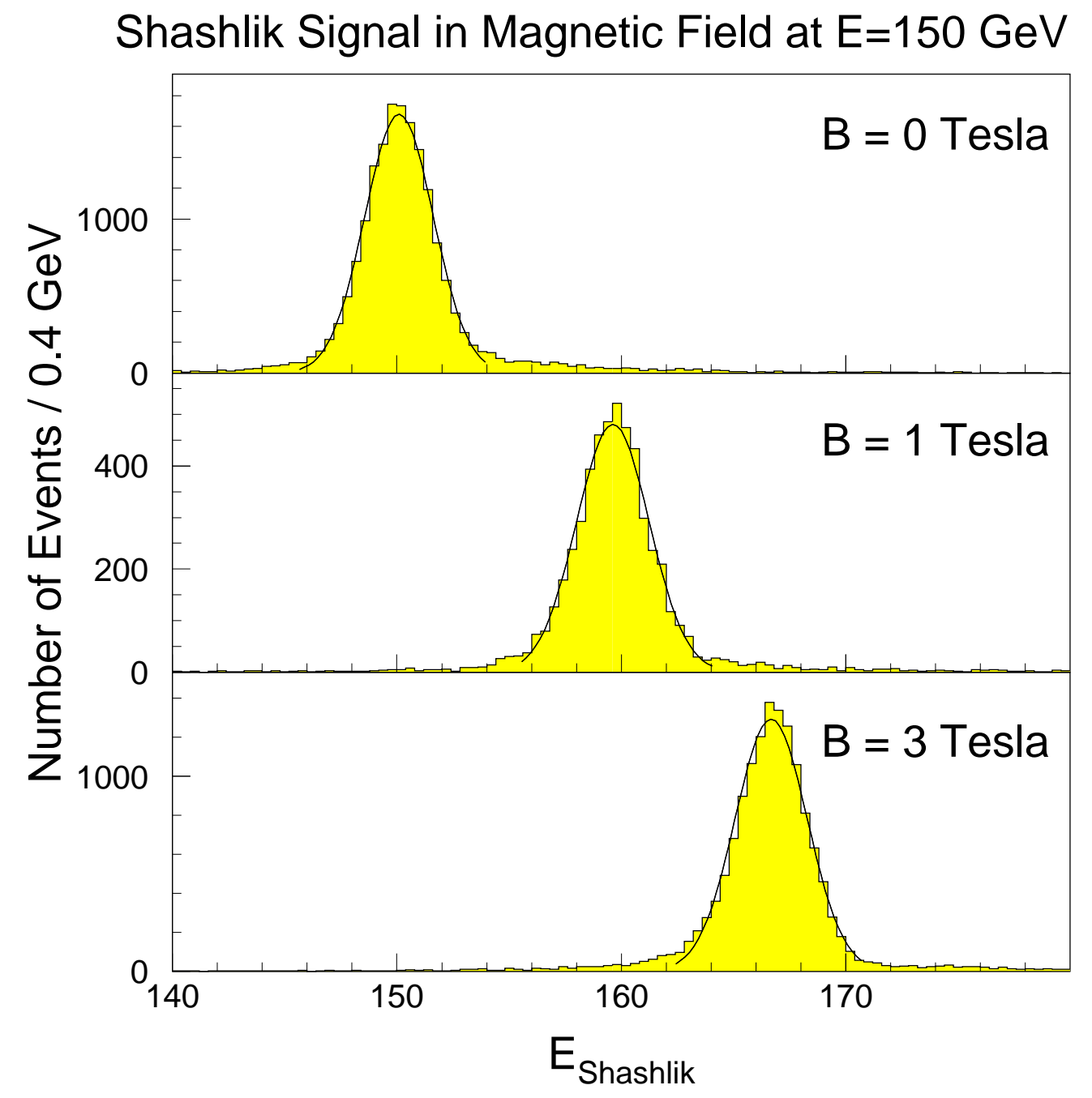

Figure 3: Energy distributions for $150 \mathrm{GeV}$ electrons, for $\mathrm{B}=0,1$ and 3 Tesla without preshower detector. The calibration constants are kept to those values obtained at 0 Tesla. 


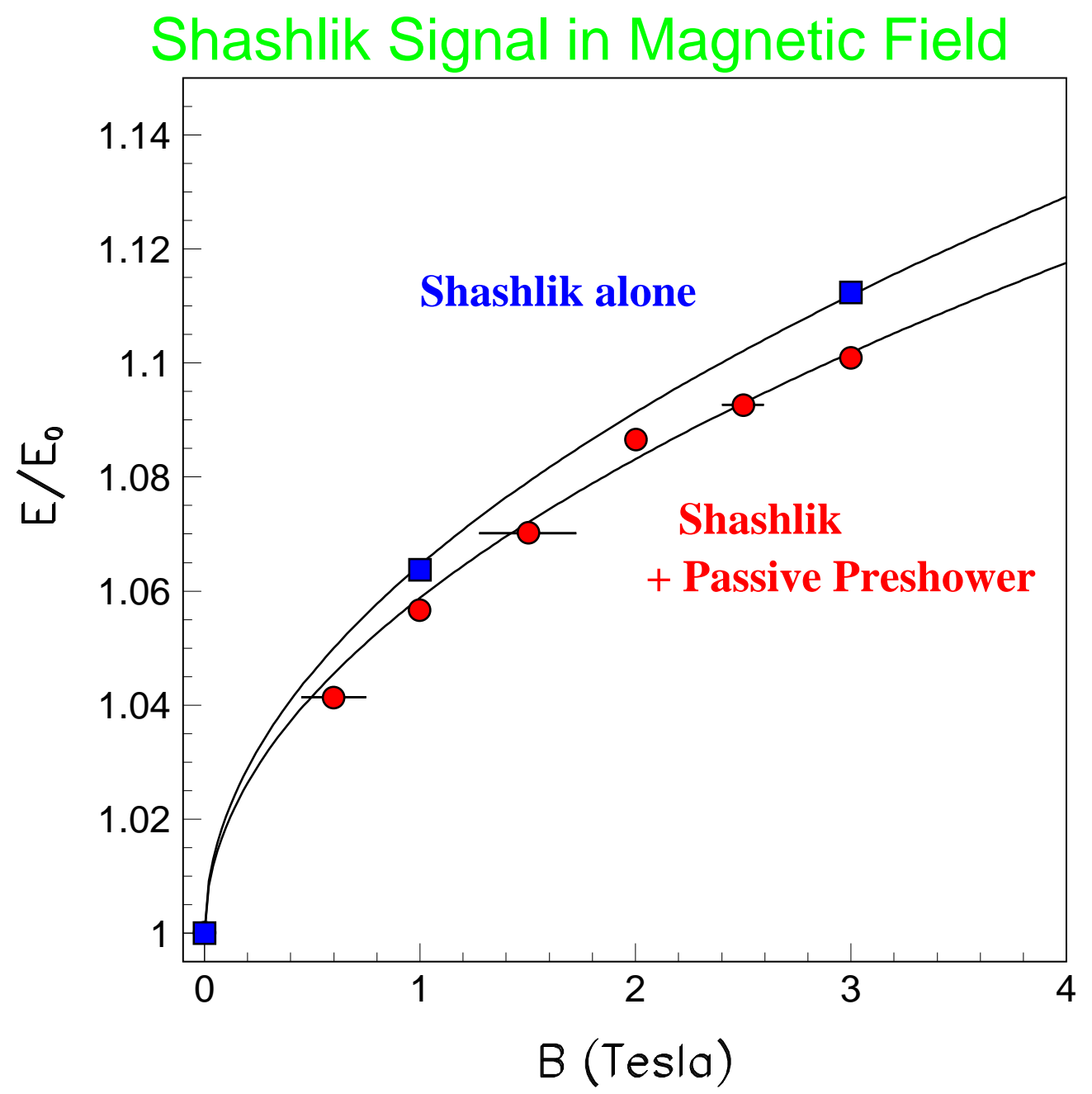

Figure 4: Shashlik response for $150 \mathrm{GeV}$ electrons in the magnetic field for Shashlik alone and with passive preshower detector ( $3 X_{0}$ material in front of Shashlik). The curves are to guide the eye. 
$\mathrm{e}^{-}, 10 \mathrm{GeV}$, full simulation

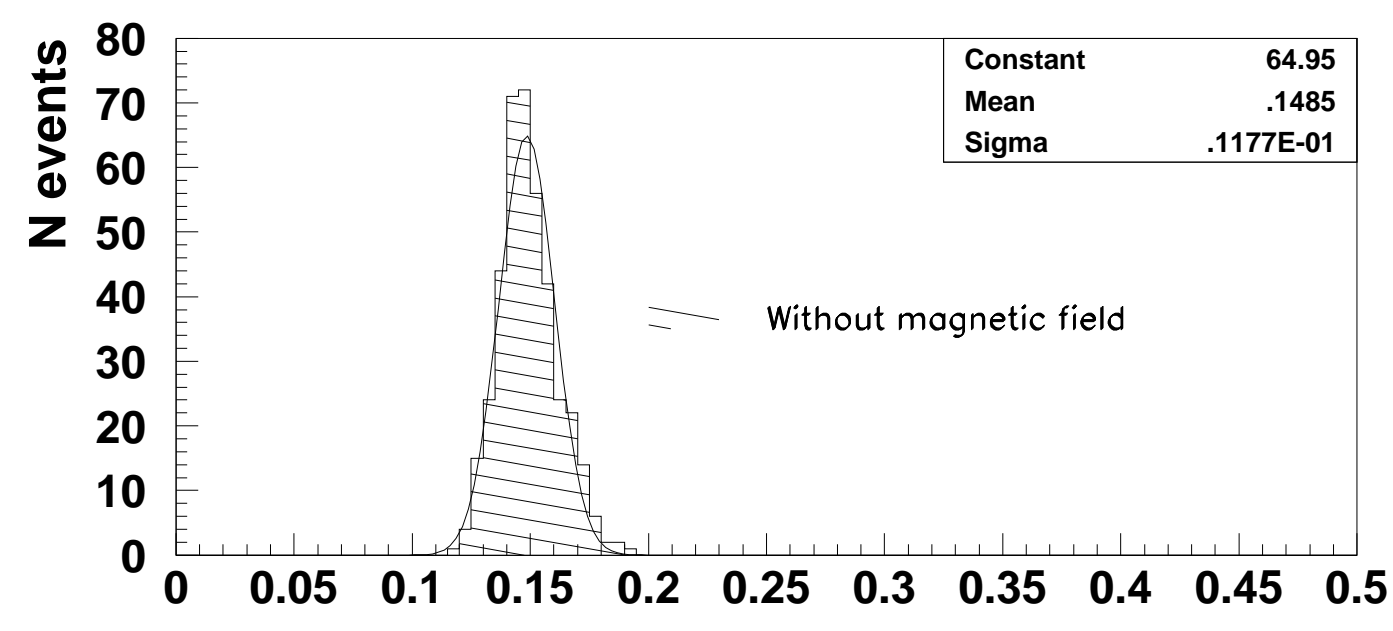

Energy in scintillator (GeV)

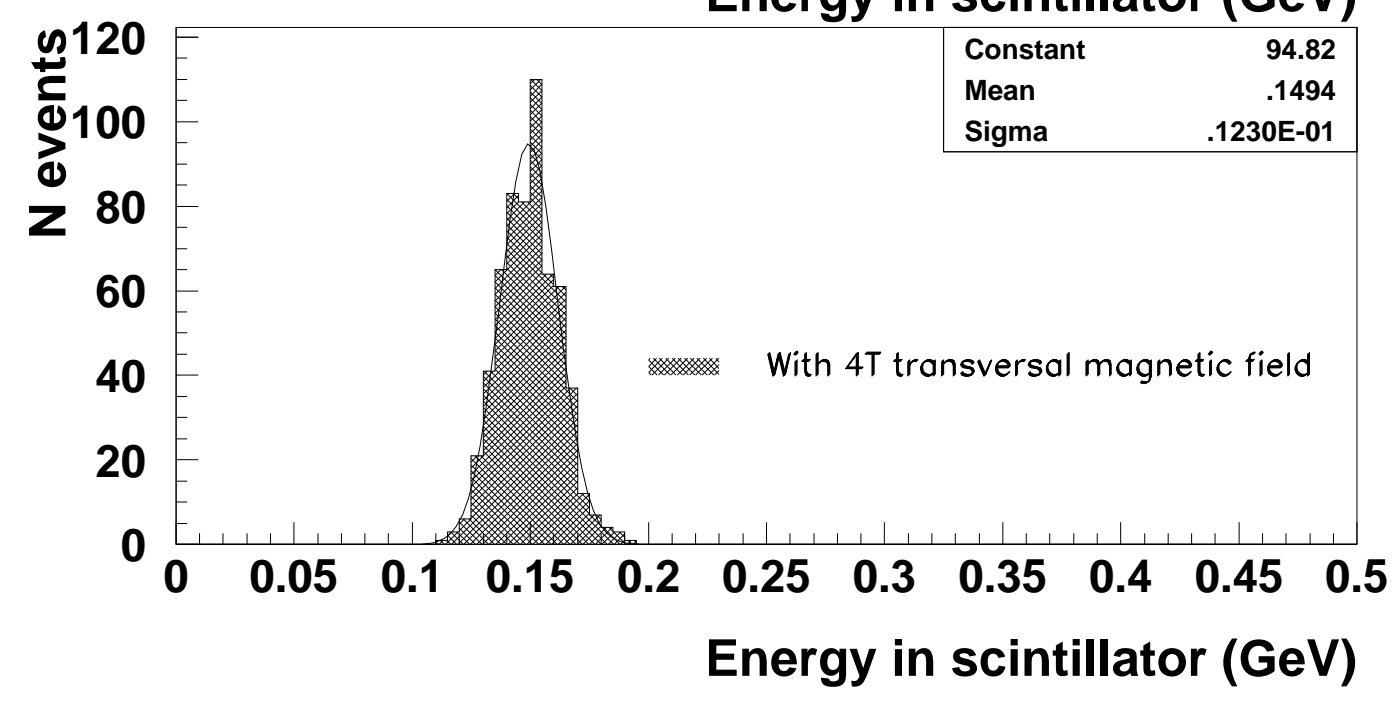

Figure 5: Energy deposited in the scintillator for $1 \mathrm{GeV}$ electrons without magnetic field and with a 4 Tesla transversal field, as predicted from Monte Carlo simulation. 


\section{Shashlik Projective - Linearity}

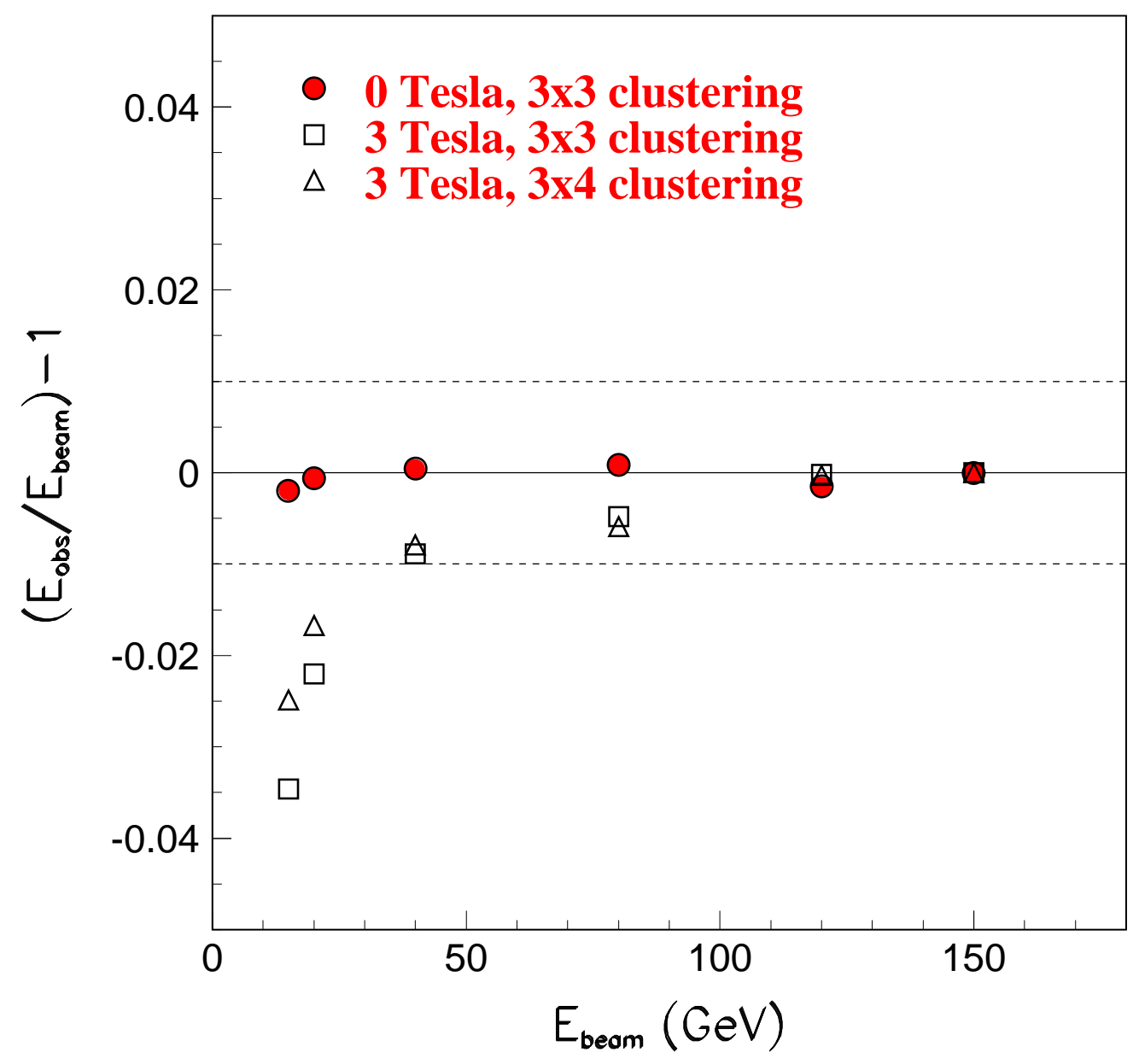

Figure 6: Shashlik signal linearity at 0 and 3 Tesla. Data are normalized at $150 \mathrm{GeV}$. 


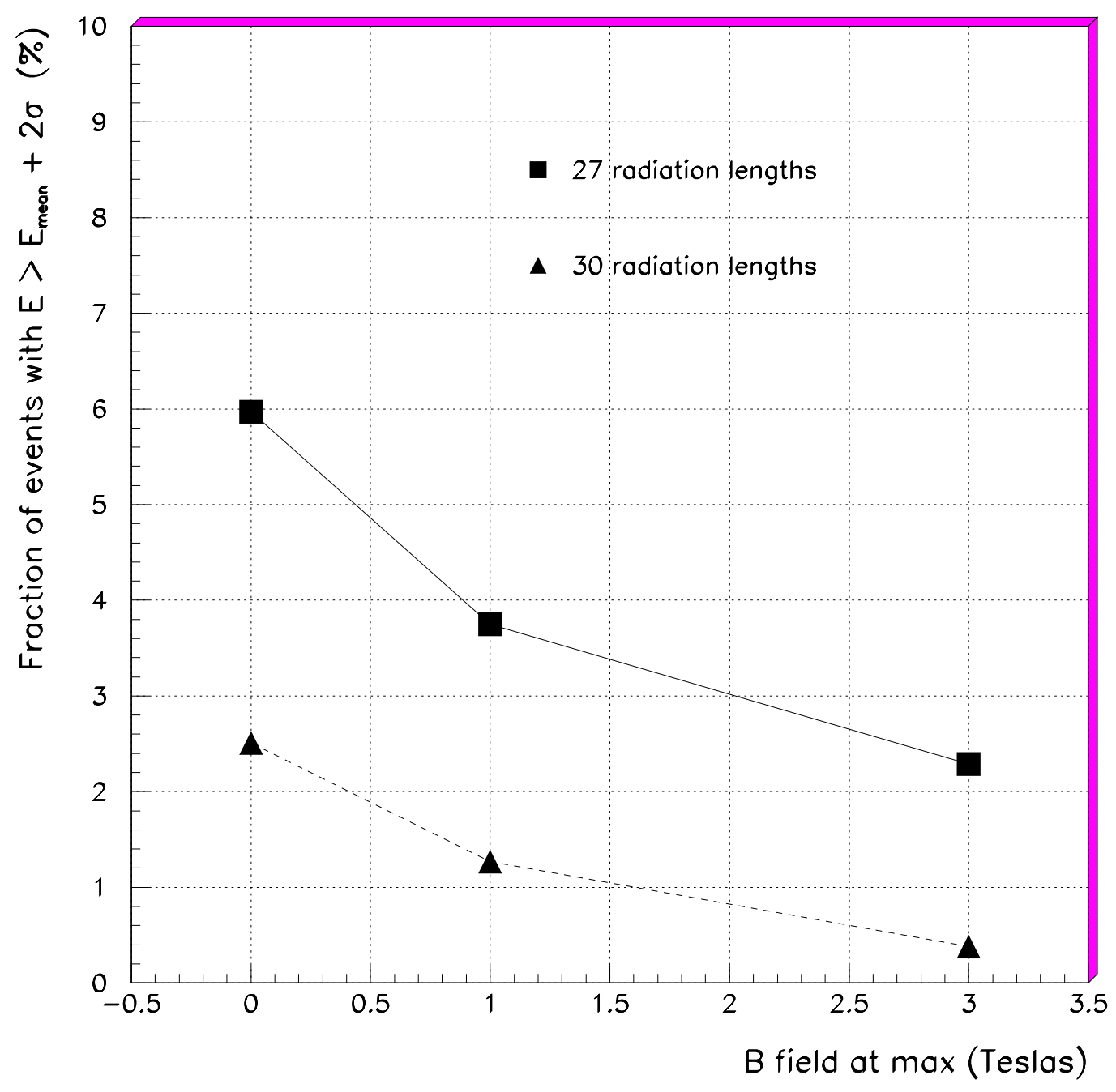

Figure 7: Excess of high signal events as a function of the calorimeter depth and of the magnetic field intensity. The excess is defined as the fraction of events having a signal greater than the Gaussian peak +2 sigma. The points at depth equal to $30 X_{0}$ correspond to data with preshower detector in front. 


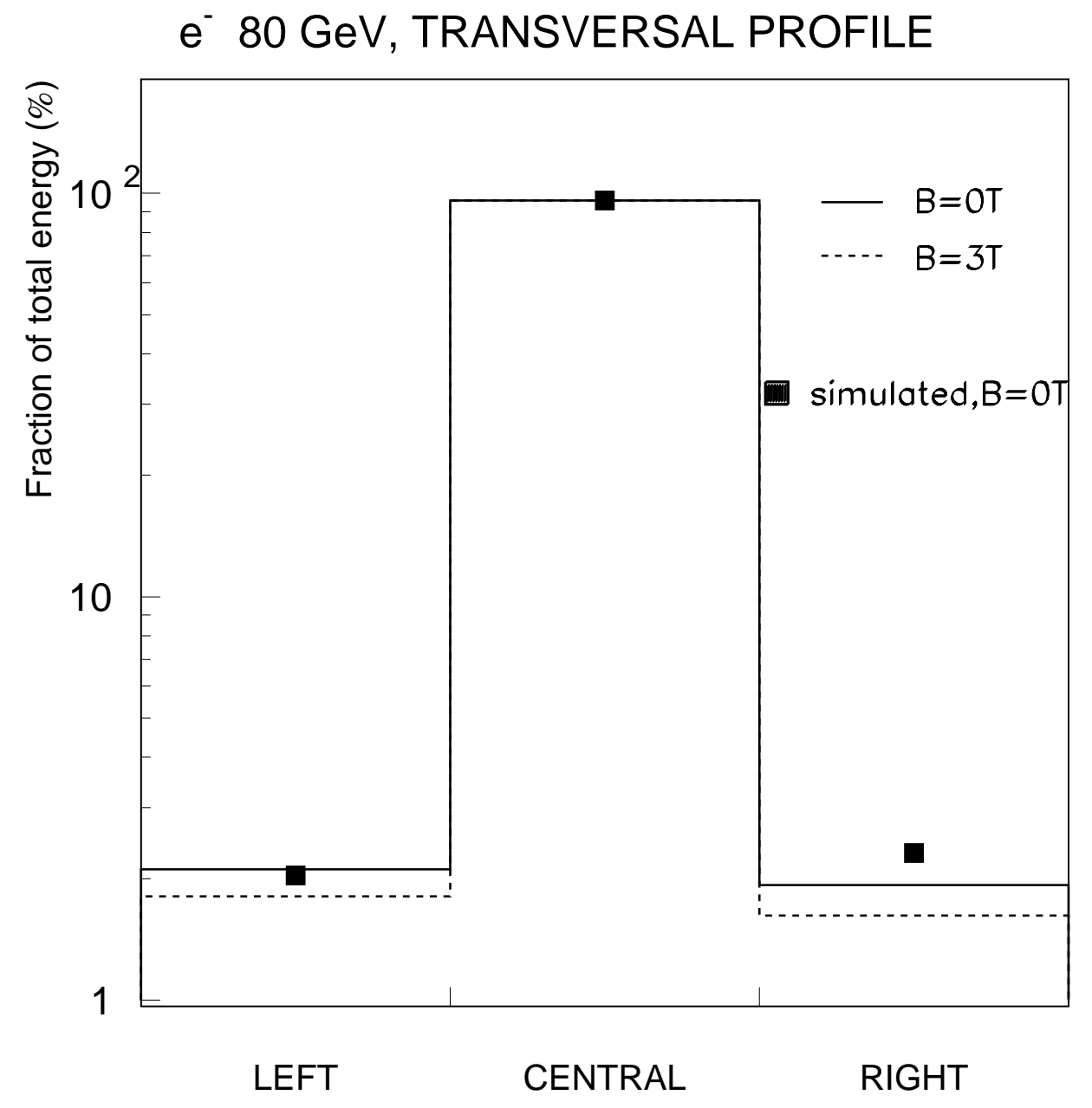

Figure 8: Transversal profile in the field direction for $80 \mathrm{GeV}$ electrons. Square black points give Monte Carlo simulation without field. Energy is normalized in \%. 


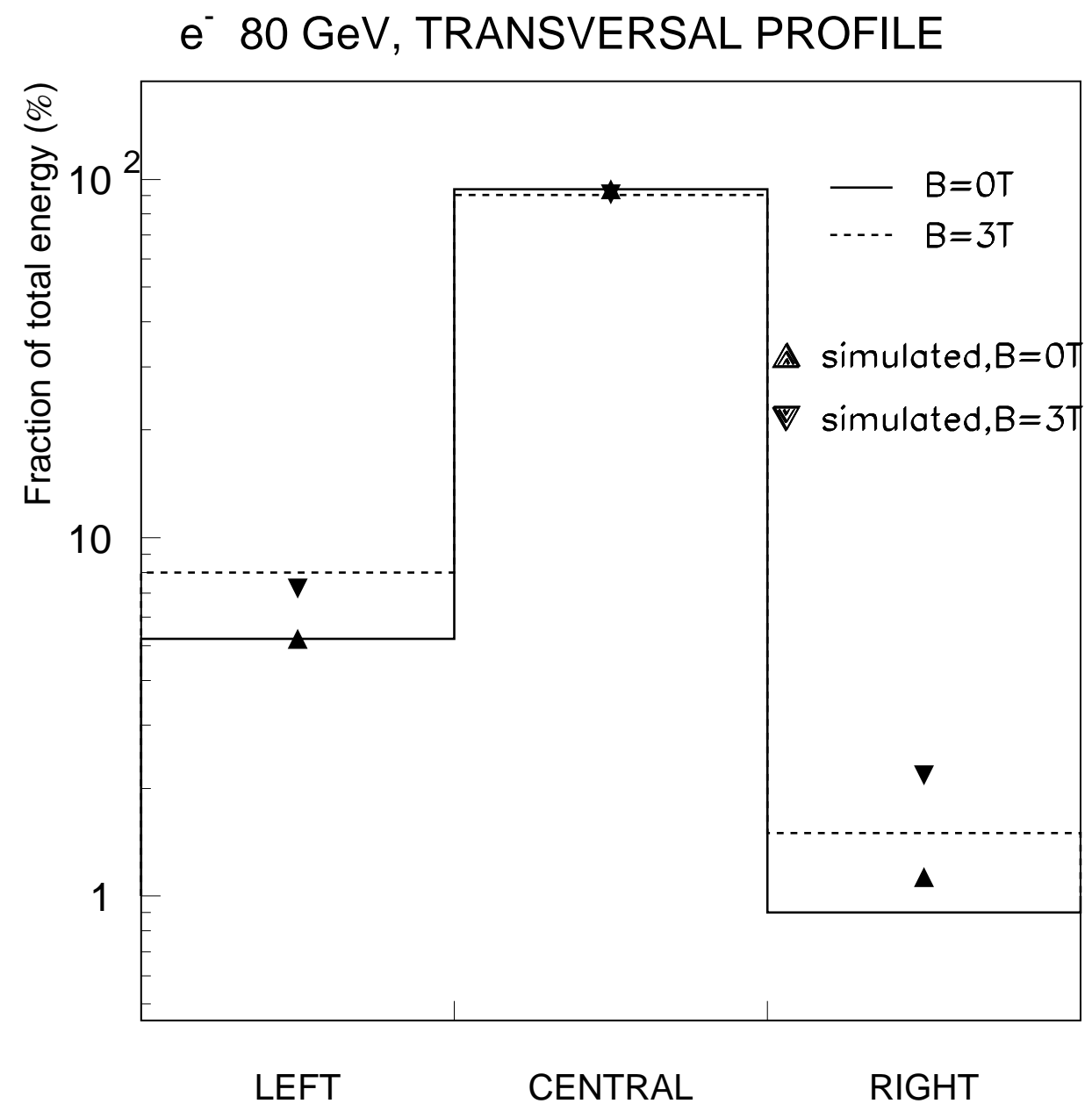

Figure 9: Same as the previous figure, but for in direction perpendicular to the field with and without magnetic field. Also shown are Monte Carlo predictions with and without field. 


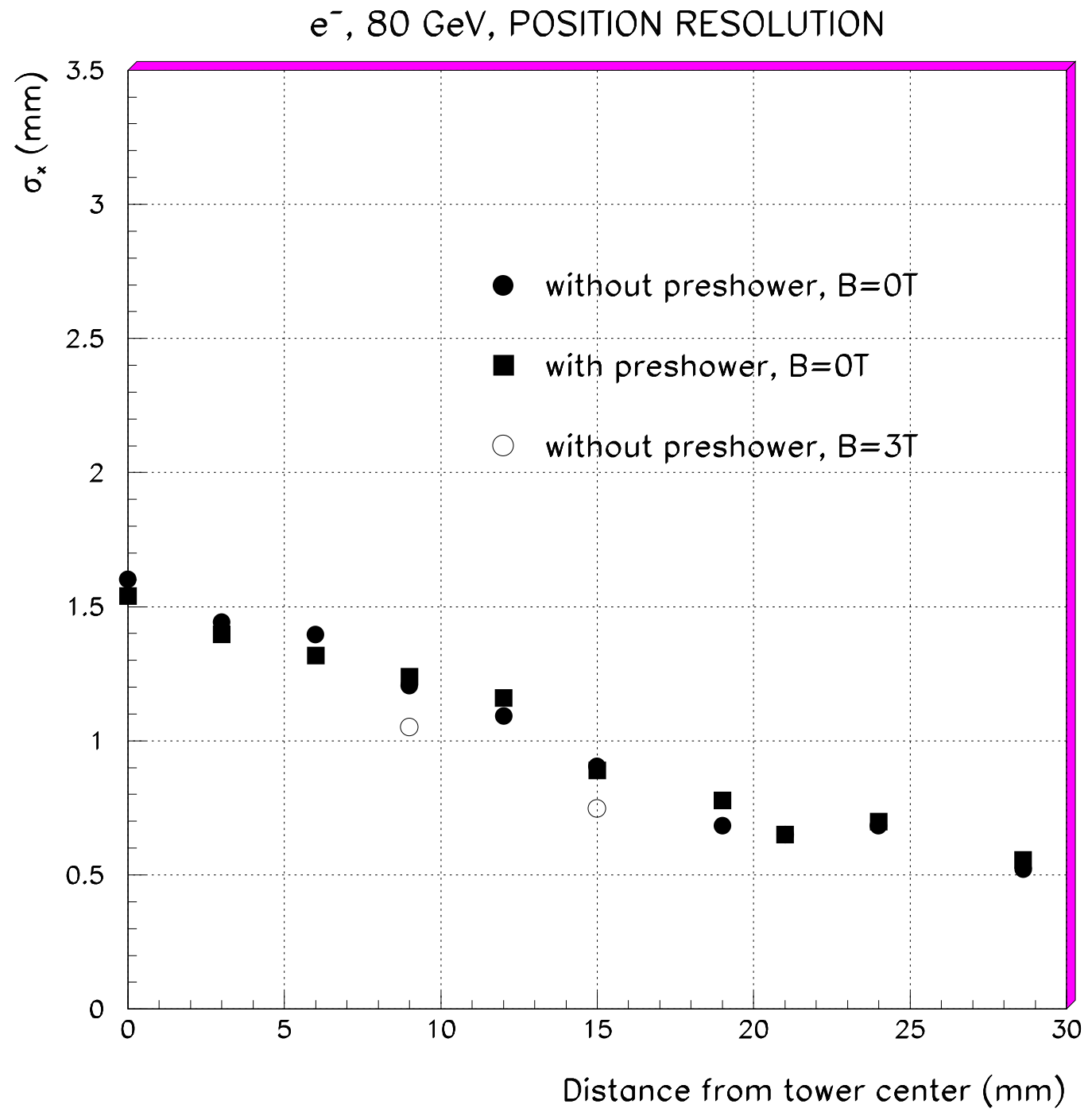

Figure 10: Position resolution for $80 \mathrm{GeV}$ electrons as a function of the distance to tower center with and without magnetic field. 


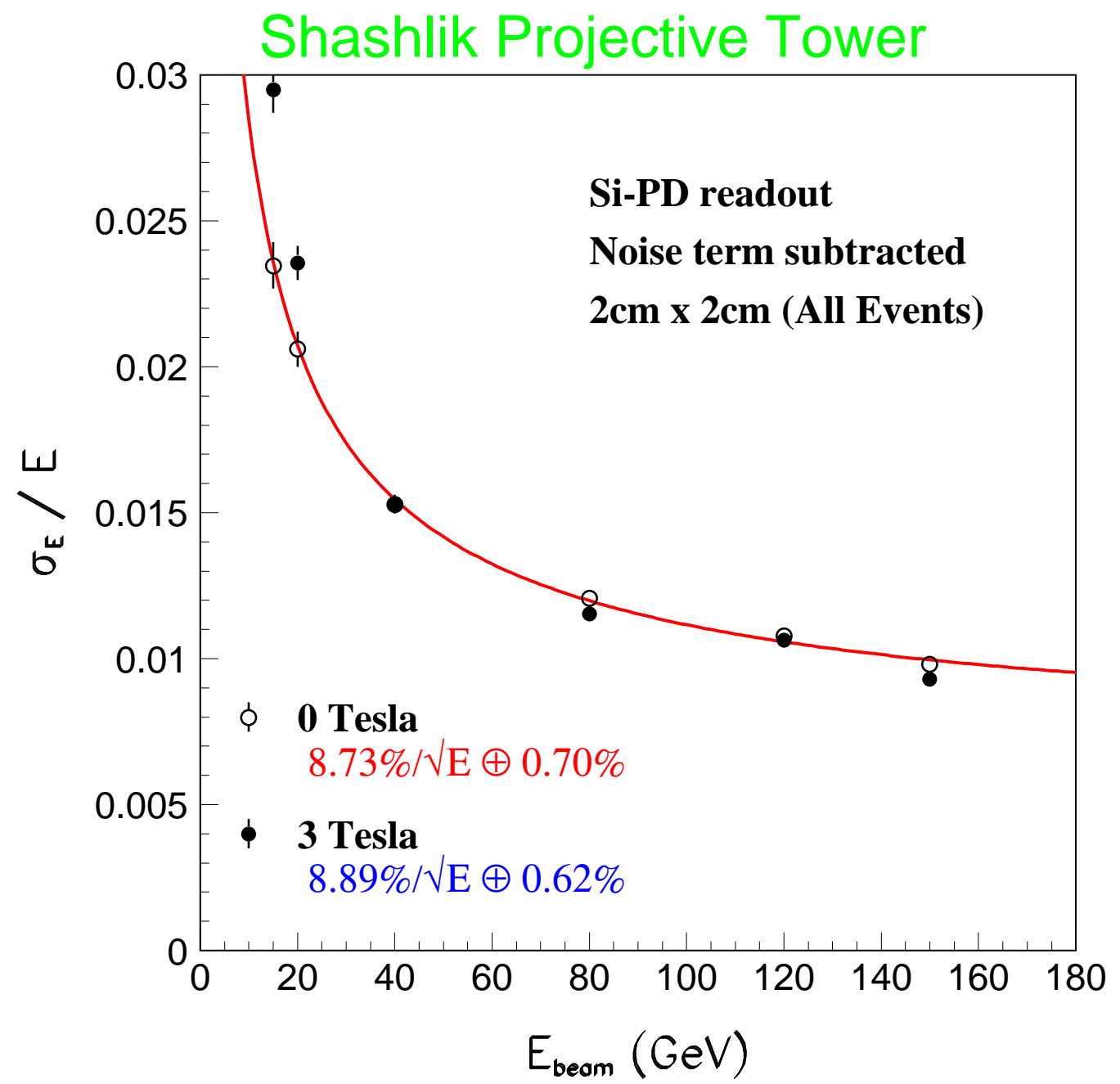

Figure 11: Energy resolution of Shashlik alone in 0 and 3 Tesla. The curve is the result of the fit for 0 Tesla. 


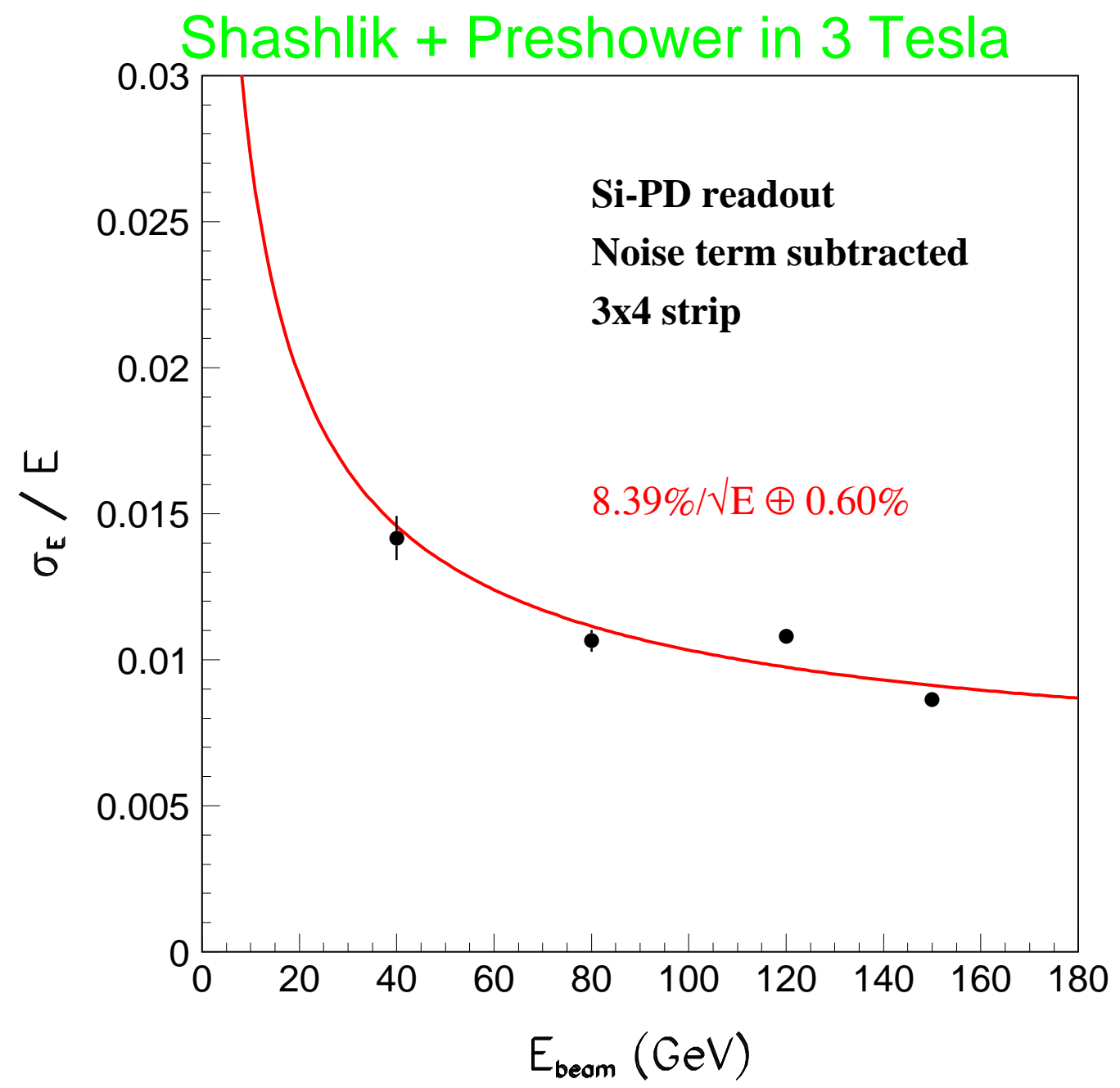

Figure 12: Energy resolution of Shashlik plus preshower detector in 3 Tesla magnetic field. 